\title{
RODRIGO FACIO Y SU GENUINO PENSAMIENTO HUMANISTA EN LA UNIVERSIDAD
}

\section{RODRIGO FACIO AND HIS GENUINE HUMANIST THOUGHT IN THE UNIVERSITY}

\section{Emmanuel Cerdas Prado*}

\begin{abstract}
RESUMEN
Este ensayo consiste en estudiar el pensamiento humanista del Rodrigo Facio desde la perspectiva de un estudiante. En este sintético ensayo se explica su aporte a la vida universitaria, a la concepción de la educación superior y como estatista en la constituyente de 1949, la cual ha marcado la vida de los costarricenses. En conmemoración de sus cien años de nacimiento, se le da la oportunidad a un estudiante, en un certamen de ensayo, de profundizar en su humanismo para definir desde los tiempos recientes el baluarte de aquél hombre al modelo universitario actual. El sistema universitario necesita tener como referencia para las personas jóvenes figuras como Rodrigo Facio, fomentando la transmisión académica de una juventud activa e interesada por la realidad del país.
\end{abstract}

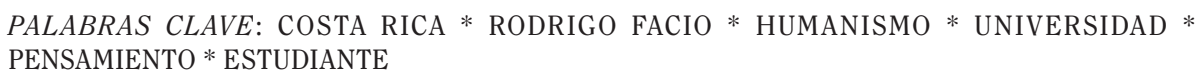

\section{ABSTRACT}

This essay consists in studying the humanistic thinking of Rodrigo Facio from the perspective of a student. In this syntetic essay it is explained his contributions to universitary life, the conception of education superior $y$ as a statist in the constituent of 1949, which has marked the life of Costa Ricans. In conmemoration of his hundred years of born, the opportunity is given to a student, in a literary contest, of deepen into

Cursando la carrera de Ciencias Políticas de la Facultad de Ciencias Sociales de la Universidad de Costa Rica. Ganador del concurso estudiantil de ensayo celebrado en conmemoración del centenario del natalicio de Rodrigo Facio (2017).emmanuel.prado1099@gmail.com 
his humanism to defined from our recent times his actions for universitary model. The university system needs to have as a reference for young people, figures like Rodrigo Facio, promoting the academic transmission of a participating youth and interested in the reality of the country.

KEYWORDS: COSTA RICA * RODRIGO FACIO * HUMANISM * UNIVERSITY * THOUGHT * ESTUDIANTE

\section{INTRODUCCIÓN}

Adentrarse en el pensamiento de Rodrigo Facio no es en absoluto una tarea fácil: es sencillo por supuesto señalar su mayor proyecto de vida, la Universidad de Costa Rica, como institución baluarte de la educación, sin embargo es ineludiblemente complejo identificar de forma numérica, el espíritu material en su obrar dentro de la institución y aún más allá, los señalamientos que realizó acerca de Costa Rica en sus libros - por mencionar uno de sus aportes en literatura económica- sobre la Banca Estatal; dada su variada cantidad. En este mismo orden de ideas, sus aportes en infraestructura, planificación y desarrollo de la teoría económica se quedarán en este ensayo a un lado, ya que donde más importancia resguarda su legado es en su sentido humano y en la involucración de la Universidad con el accionar de la realidad nacional. Rodrigo Facio Brenes, un hombre de hacer, pensar y dejar sobre su obra más pensamiento — como en este significativo ensayo-; es una personaje "inoxidable" que a pesar de las décadas, será recordado a causa de su gran legado.

Muchas discusiones se han generado alrededor de Facio, sin embargo, una que es pilar de nuestro modelo universitario y que ha estado oculta por varios años de forma "parcial", es la importancia otorgada a la Reforma de Córdoba, en la constitución de la autonomía universitaria y pensamiento crítico. Asimismo, se debe dar lugar a la comunidad estudiantil, que como bien es conocido, ha cumplido en Costa Rica un papel primordial en los movimientos sociales cuando el país así lo ha requerido. Me atrevo a exponer, que el movimiento estudiantil universitario, es la estructura de movilización que frente a acontecimientos coyunturales, ha dado más asistencia al ejercicio de la protesta. Esto fue impulsado por el mismo Rodrigo Facio en su discurso de despedida de la Rectoría en el año 1961, en el cual menciona en donde deben estar los estudiantes y cual es su labor en la articulación social. Si bien no dijo en forma literal, se ha extraído la idea principal de una de sus frases más recordadas: "Los estudiantes deben estar en las aulas pero cuando las condiciones del país lo ameriten deben estar en las calles" (Facio, 1961), así se popularizó y desde entonces ha sido uno de las consignas de la población estudiantil.

Sin embargo, no es fin propio, como mencioné anteriormente, dedicarme a hacer una mirada ortodoxa, osada y reciclada de quien es Rodrigo Facio y cuál ha sido su obra en la Universidad, sino más bien, desde la mirada estudiantil se planea representar por medio del ejercicio del ensayo su preciso y más importante aporte a la Universidad; el legado de un estudiante, docente $y$ forjador de una universidad a través de su pensamiento.

\section{HUMANISMO UNIVERSITARIO}

Partiré desde un borde quizás olvidado, pero que cuando se lee se sienten los rubores del orgullo alzarse. Me refiero a un escrito que el mismo Rodrigo Facio Brenes dedicó a la Revista de Filosofía titulado: "Dos Palabras sobre la Revista de Filosofía de la Universidad de Costa Rica”. En este detalla la necesaria y simbólica aparición de la mencionada revista, en el acontecimiento extraordinario de los días donde se acababa de inaugurar la Facultad de Ciencias y Letras, hoy conocida como Estudios Generales. Expresa Rodrigo Facio que esta 
hazaña corresponde a un éxito para el Departamento de Estudios Generales, ya que tiene como objetivo "darle al estudiante una visión general del mundo" (Facio, 1957, p.3) y profundizar en la filosofía por medio del ejercicio de la razón.

Estudios Generales, que apenas se constituía como un Departamento por aquella época en la que Rodrigo Facio era Rector de la Universidad, venía a entregarle al estudiante una semilla sobre el humanismo que se desarrollaba en otras partes del mundo, lo sentaba a reflexionar y lo aguardaba en el interesante mundo del pensamiento denominado contemporáneo. Así como denominaba el ejemplar rector: "la inclusión de los Estudios Generales en los programas universitarios (...) responden al renacimiento de la Filosofía y al reconocimiento cada vez más explícito de su necesidad" (Facio, 1957, p. 3). Las inquietudes que deja ver en la publicación sobre la revista, apuntan a que tenía toda su fe en una comunidad estudiantil más viva, en una juventud con mayor pensamiento en el futuro.

Asimismo, es verdaderamente elocuente al exponer más adelante en este escrito, cómo define a su grupo de compañeros directores o bien, funcionarios de la universidad; iniciando por el historiador Rodolfo Mondolfo llamándolo como un campeón de "la investigación intrépida de la verdad, libre de todo prejuicio" (Facio, 1957, p. 4). A otro colega relevante, como lo es Constantino Láscaris, lo felicita por sus generosos esfuerzos por acoplarse a nuestra cultura y cuenta sobre los cursos que impartirá en la Universidad; además de la significativa tarea que está realizando por comprender el pensamiento costarricense y lo despide de su escrito deseándole buenos augurios. Continúa - aquí brevemente solamente lo mencionaré- rescatando en forma de despedida una sección de crónicas, donde menciona los antecedentes filosóficos de la Universidad de Santo Tomás y reflexiona sobre los acontecimientos que en buena o mala hora, nuestros antepasados han realizados y menciona que el reconocimiento de lo hecho por nuestros antepasados es justo, “(...) porque ninguna generación hace al mundo, sino solamente la prosigue" (Facio, 1957, p. 5). Estas palabras que de manera emocional calan profundamente en mi pensamiento, son palabras célebres que han sido olvidadas entre la creencia sobre la acumulación de méritos físicos y didácticos, pero detrás de un pensador como el que disponemos a analizar aquí, podemos descubrir que existen entre los escritos palabras tan monumentales que resguardan per se la esencia de una persona.

\section{LECTURA DE LA REALIDAD NACIONAL}

El arduo trabajo que realiza Facio también es verificable en su vida como estudiante, específicamente en la realización de la prosa dirigida en el ensayo, la cual se refiere a su recordado trabajo Estudio sobre la Economía Costarricense, que comprende un repaso del actuar costarricense en cuanto a la solución de problemas económicos que el mundo internacional mira en las problemáticas en el desarrollo económico de la Costa Rica de ese entonces, sus pobladores tenían dentro de sus preocupaciones el tema de la economía. El trabajo es presentado por Facio en octubre de 1941 para optar por la Licenciatura en Leyes, en la Facultad de Derecho de la Universidad. En el estudio podemos hallar sus principales hallazgos sobre su pensamiento convincente $y$ decidido, con esto solamente haré referencia al apartado que aparece luego de la nota de introducción, donde Facio explica de forma vehemente y necesaria el tema de la economía como un factor primario dentro de los temas irresueltos. En una profunda pausa que analizaremos: “... sufre el país actualmente diversos males de índole económica y social que comprometen la estabilidad de su régimen" (Oduber, 1994, p.144). El mensaje es conciso y directo, el deber nuestro es Costa Rica, sino ¿de dónde serán los próximos próceres de la patria? y no quiero caer en el discurso nacionalista, sino más bien devolver en la memoria, frente a la necesidad de actores inmediatos precisamente haciendo lo que se les espera que hagan: actuando. Es más propenso enfrentarnos al menosprecio de muchos antes que vernos construyendo país; solo que una vez lo construyamos, el "menosprecio" será propenso no en nuestros zapatos. El mensaje de Facio 
sobre su auténtico y repetitivo legado, que sea cualesquiera que verse sobre él, nos hace un manifiesto llamado a apropiarnos de nuestro país; sobre todo a la juventud desentendida, podemos apreciar a través de su pensamiento el profundo humanismo que gira en torno del 'otro', hacerlo parte de la lucha, para luchar con él.

Continuamos con la nota que escribe Facio, donde expone que no es únicamente tema de los estudiantes "preocupados" el tener injerencia en los asuntos nacionales, más — de forma ingeniosa- expone que ellos son también encargados de "(...) estudiar, de adelantar puntos de vista, sugerir". Es decir, el deber de la academia lo conserva en su esplendor, exponiendo la necesidad de poseer figuras académicas no solamente joviales sino juveniles que emprendan su camino al conocimiento científico. Cuando finalmente concluye su nota, expresa que el estudio está inspirado en el Centro para el Estudios de Problemas Nacionales y argumenta que es una manifestación de su generación y finaliza — de la forma más auténtica para exigirle un lugar a su generación- "Déle, siquiera eso, el valor para nuestra incorporación profesional" (Oduber, 1994, p. 144). Siguiendo el orden del análisis previo que realizamos sobre el estudiante en Rodrigo Facio, podemos identificar la fortaleza con la que en esa frase final, no solo da un mensaje de significancia a su escrito, sino también denuncia una problemática de menosprecio que con el paso de las décadas aún podemos ver presente en nuestros centros educativos. La subestimación del estudiante. En esta línea, es rescatable ver la entrega adicional al trabajo que estuvo realizando en el Centro para el Estudio de Problemas Nacionales; aquél espacio intelectual compuesto por jóvenes organizados, destacó en su futuro como un heredero del Centro Germinal (creado en 1912), que al igual jugó un rol importante con grandes intelectuales de nuestro país, como Omar Dengo por ejemplo, y en su naturaleza dirigida por figuras humanistas.

\section{DOCENTE Y ESTUDIANTE}

En el acercamiento por comprender el pensamiento de Rodrigo Facio es necesario identificar el carácter que desarrolló como maestro de su casa de estudios universitaria y cuáles fueron sus postulados ideológicos, en cuanto a la ejecución de su pensamiento político. Frente a esto, en un libro escrito por Raúl Hess E:, titulado Rodrigo Facio. El Economista, analiza todo un recorrido de la vida de Rodrigo Facio y diversas etapas de su vida fundamentales en su pensamiento y obrar humano. Hess, quien en el año 1951 tuvo el privilegio de ser alumno de Rodrigo Facio en su tercer año de carrera, nos presenta unas observaciones sobre cómo era su forma pedagógica de emprender la enseñanza. Nos menciona que para nada, sus clases eran de forma magistral, sino que estaba presente su "innata vocación de pedagogo" (Hess, 1972, p. 142), cualidades descritas por él como simpleza y claridad en la exposición. Adicionalmente, comenta que era costumbre suya repasar la materia luego de haber explicado la teoría, esto mediante interrogatorios a algunos estudiantes cuya respuesta simple era el mayor medio, ya que si no se respondía era un indicio de que no se dominaba la materia frente a toda la clase. Comenta Hess, que varios estudiantes no llegaban a clase por el miedo de no saber las respuestas de sus interrogatorios. Sin embargo, esos eran los espacios propicios para resolver preguntas sobre el tema y este era el acierto de sus lecciones, caracterizadas por una "permanente jovialidad" que generaba un diálogo entre docente $y$ estudiante. Es tan convincente este hecho, que el profesor Facio desarrolla una humildad ejemplar, que a más de un docente en la actualidad le hace falta frente a los estudiantes: cuando algún alumno le indicaba algún señalamiento erróneo sobre la materia impartida, este hacía una breve pausa y le agradecía su respeto en cuanto a la observación. Estos rasgos de virtud en su clases también fueron visibles entre los compañeros de trabajo en la Escuela de Ciencias Económicas, denotadas por su solemne apoyo a los cambios en Planes de Estudio para la carrera, donde siempre brindaba su apreciación personal en apoyo a reestructuraciones en beneficio del conocimiento científico $y$ humanista del estudiantado.

Otros factores que muestran su humildad eran sus llegadas a la universidad cerca del barrio González Lahmann en taxi y cuando fue 
alumno de nuevos cursos impartidos en la cátedra sobre Desarrollo Económico en 1956, donde con "cariño y sincero respeto" fue alumno de Raúl Hess, quien nos regala estas breves anécdotas y según él contempla: "(...) siempre vivió una permanente actitud juvenil".

\section{IDEOLOGÍA EN RODRIGO FACIO}

Para el pensamiento de Rodrigo Facio, que es amplio en sus visiones y convicciones, la identificación de su ideología político-económica ha sido la gran dificultad de estudio, ya que en nuestro siglo xxi, la simpatía con cualquier tendencia es prejuiciosa y no se quedará fuera de nuestros lectores y lectoras la apatía política. Pero para nuestro pensador que era en suma un animado precursor de su doctrina, era necesario un modelo de Estado y este fue el que defendió.

El 29 de octubre de 1957, se celebró una conferencia que fue brindada por Rodrigo Facio sobre las escuelas políticas y económicas, encasillada en un título denominado "La Filosofía en el Siglo xx"1, celebrada en la Escuela de Educación. Dentro de las principales ideas que aborda al inicio de su conferencia, es tratando el liberalismo y el socialismo. Explica que lo lamentable de ambas doctrinas es que no tienen aún sensibilidad al usar al hombre como medio para otros fines y que cada vez más, ya no se busca el beneficio de un mayor número de personas. Además, menciona que los marxistas no visualizaron de manera idónea a la sociedad en general, al someterla a una decadencia del capitalismo: “(...) se puede afirmar que en Occidente está en formación (...) una nueva concepción económica y social de carácter pragmático, realista $y$ flexible, en donde rasgos capitalistas se mezclan con rasgos socialistas y matices individualistas y liberales" (Hess, 1972, p. 162).

En este pensamiento, él asegura no solamente que esta concepción nueva ocurre en Occidente, si no también en Costa Rica, donde

1 Los comentarios sobre la conferencia han sido recogidos de igual manera del libro Rodrigo Facio El Economista del autor Raúl Hess E. y del artículo publicado en la Revista de la Universidad de Costa Rica por Rodolfo Cerdas en 1960. ciertamente estos rasgos se han constituido de forma beneficiosa como parte de nuestra vida democrática. La ideología de Facio va muy ligada a esta aceptación mixta entre capitalismo-socialismo en la búsqueda implacable de la dignidad humana, siendo esta última el eje principal de cualquiera de las dos doctrinas. Por otro lado, Facio afirma que:

Los países sub-desarollados, si no se hace algo realmente imaginativo $y$ audaz por parte de los países dirigentes y de los organismos internacionales, para garantizarles pan y libertad. (...) pueden, con suicida impaciencia, echar mano de la sencilla y prometedora fórmula comunista (Hess, 1972, pp.162-163).

En esta frase, Facio enfatiza en la necesidad de que los organismos internacionales intervengan mayormente en el servicio de países sub-desarrollados, y expone el tema del comunismo como una mala vía, e incluso suicida, que 'privaría' la libertad. Podemos calcular que el enfoque con el que Facio se encarga de medir esta clase de regímenes, tiene que ver más con panoramas económicos que con panoramas sociales, ya que en comparación, las diferencias entre ambos son abismales, sin embargo, podemos también contextualizar que la popularidad de los organismos internacionales para la época en la que se desarrolla esta conferencia (1957) habían desarrollado una gran y exitosa labor.

Alrededor de este escrito, se reveló gran controversia por los grupos de izquierda, uno que sin duda generó disturbios fue un artículo publicado en la Revista de la Universidad de Costa Rica en el año 1960 por el autor Rodolfo Cerdas Cruz, en un curso de Filosofía del Derecho. En el capítulo iv, reconoce las aseveraciones realizadas por Rodrigo Facio, las principales son "(...) la de que el triunfo del socialismo en los países de hoy de democracia popular, es un triunfo antimarxista; $y$ por otro lado la solución que el Sr. Rector le da a los problemas sociales" (Cruz, 1960. p 104). Dado el carácter de nuestro estudio, se dará a la tarea de identificar las respuestas que generó en Rodrigo Facio, através de una carta enviada al profesor de Rodolfo Cerdas, sobre las observaciones del entonces 
joven crítico. Responde Facio acerca del triunfo de las revoluciones con tinte "marxista", que la madurez alcanzada por el más grande acontecimiento revolucionario de la época, es decir, la Revolución Rusa, no se encontraban en los estados de producción industrial en condiciones de competir. El argumento presentado por el joven Cerdas hace referencia a la consolidación de los Estados donde el proletariado está en el poder, es decir, que son definidos como "(...) la conversión del capitalismo existente en el país al capitalismo de estado; $y$ la segunda etapa es la transformación del capitalismo de estado socialista" (Cruz, 1960. pp 105-107). Ante esto, la respuesta de Facio es “(...) de acuerdo con el materialismo dialéctico e histórico, que constituye la filosofía fundamental del marxismo, (...) ¿No constituye, por acaso, la explicación ofrecida por el joven estudiante, el reconocimiento de una nueva falta de cumplimiento de las, para el marxismo, leyes "inexorables de la historia?"”. La respuesta de Facio acude a la profundidad del análisis de Estado que se resolvió en la respuesta común de países de este corte, especialmente China $y$ unss. Sin embargo, ante las seguidas afirmaciones de que en Occidente se desea consolidar un Estado capitalista, Facio resuelve concluir con su afirmación sobre la mezcla de rasgos capitalistas y socialistas previamente expuesta:

Cuál será el régimen que salga de esa política, cuál será su denominación, podrá ser preocupación de los dogmáti$\cos y$ doctrinarios, pero no mía. A mí no me desvela cómo se llamará el régimen que resulte de esa política, si dentro de él se respeta la dignidad del hombre y se le reconocen sus libertades espirituales $y$ políticas esenciales, $y$ se le garantiza un elevado y creciente nivel material de la vida (Hess, 1972, p. 165).

Por lo tanto, podemos deducir que esbozar en un planteamiento sobre las posturas ideológicas de Rodrigo Facio es estrictamente difícil, pero que a partir de lo previamente mencionado, resalta por encima de su ideología el tema de la dignidad humana, postulados planteados del nuevo humanismo que vienen a conservar en la persona su estabilidad y libertad humana a nivel individual. En esta misma línea, en el obrar de Facio y en relación con su juventud podemos apreciar que tuvo unos primeros años que prestigiaban al socialismo $y$ eso marcó su conducta, por lo tanto de forma pública será imposible hallar información en su etapa adulta que nos permita plantear su ideología contraria al socialismo.

\section{CONSTITUYENTE DE 1949}

Parte de los hechos más reconocidos de Rodrigo Facio, fue su protagonismo al representar sus posicionamientos progresistas en materia de banca estatal y en sus intervenciones como oratoria en la elaboración de la constituyente de 1949. Es comentado por los estudiosos de la constituyente, que el papel de Facio fue de los más deslumbrantes escenarios de aquella época. Importante dedicar aquí la convocatoria que posterior a la Guerra Civil, José Figueres Ferrer nombra a una comisión especializada de nueve personas, entre las cuales en función de Secretaría General brindaba sus servicios el Sr. Facio.

A criterio propio, pese al enorme trabajo realizado por Rodrigo Facio, fue inminente su fracaso de proyecto constituyente que tantas noches se dedicó a plantear nuestro pensador. Para contextualizar, la Asamblea Nacional Constituyente estuvo integrada por 45 curules, de ellos 34 eran parte del Partido Unión Nacional, seis del Partido Constitucional y tan solo cuatro del Social Demócrata, de este último era parte Rodrigo Facio; una evidente minoría frente a los otros sectores. La Asamblea decidió acogerse a la Constitución de 1871, dado que la gran mayoría era conservadora; sin embargo, Facio no se rindió y apoyado de su partido, en contra de la mayorías de legisladores, por la vía de las mociones logró acercar lo más posible sus convicciones socialdemócratas.

El aporte a la constituyente fue dificultosamente alargado a través de todo el proceso, para Rodrigo Facio quien a la edad de 32 años era parte de los proyectos de realidad nacional más sobresalientes, fue una satisfacción completa. No es muy conversado, pero los ideales principales de Facio eran esencialmente 
revolucionarios en la época, parte de esto eran las iniciativas divulgadas por el Partido Social Demócrata. Por ejemplo, se puede destacar la consigna del Partido: "Ni pan sin libertad, ni libertad sin pan. PAN CON LIBERTAD" que así como indica Oscar Castro en su libro Rodrigo Facio en la Constituyente de 1949:

(...) consigna inspirada en el pensamiento revolucionario de Víctor Raúl Haya de la Torre. Facio, admirador del político peruano $y$ de su ideario revolucionario (...) Con alguna frecuencia, en sus intervenciones de fondo en la Constituyente, acude a citas de libros de Víctor Raúl (Castro, 2003, p. 2).

En ese orden de creencias, el fin concreto de Facio en la constituyente era garantizar no caer en el despreciado personalismo político que nos llevó a la Guerra Civil. Facio con la satisfacción de su trabajo puede asegurar, como lo hizo en uno de sus discursos:

(...) vamos a darle una nueva Constitución que responda a aquellos anhelos, para que a la par de la libertad y la democracia política, se garanticen a su vez, medidas que sirvan de fundamento material para que el pueblo pueda ejercer sus derechos políticos, sin la carga de condiciones sociales y económicas que violen su capacidad de vivir y su necesidad de vivir dignamente (Castro, 2003, p 58).

\section{UNIVERSIDAD-LATINOAMÉRICA}

Volviendo al resultado en su obrar dentro de la Universidad de Costa Rica, podemos confiar que Rodrigo Facio fue de los principales gestores acerca de las relaciones sociales de esta universidad con la comunidad en la que estuviese relacionada. En esta reflexión sentaremos como base un discurso de clausura del 21 de diciembre de 1956 con fines del cierre de año. El discurso esclarece sobre la relación entre Universidad contemplativa y Universidad activa en la búsqueda de apoyar los problemas sociales que le acontece:
Hoy, nuestras universidades $-y$ el movimiento universitario en Latino América- es en esa dirección que obra, sobre todo a partir de la lucha por la reforma universitaria librada en Argentina en 1918 deben ser libres exterior e interiormente, respetadas y comprendidas, apoyadas y estimuladas, $y$ deben saber ganarse el derecho a todo esto. (....) Todo esto explica por qué es social el signo de nuestros tiempos, $y$ porqué volviendo a lo nuestro de esta noche, una Universidad como la de Costa Rica, Universidad joven y pobre en un país joven y pobre, debe tener por norte el servicios a la comunidad (Hess, 1972, pp 155-158).

\section{CONCLUSIÓN}

La labor de Rodrigo Facio al frente de sus acciones, le mantendrá por siempre en nuestro recuerdo como todo un humanista con visión revolucionaria $y$ de enfrentar las contrariedades de su tiempo para una mejor nación. La elaboración de esta reflexión se gestó muchos años después de su existencia, a pesar de estos podemos sentir el inmenso cariño con el cual describimos sus aportes a la nación costarricense. Podemos argumentar que verdaderamente divagamos en profundizar en su pensamiento humanista $y$ sus labores en la universidad en lo que respecta su filosofía de vida, esto desde la visión de un estudiante, por tanto, el aporte a la academia de este ensayo posee el atributo de señalar aquellos elementos que considera importante la comunidad estudiantil actual de la Universidad.

Como estudiante es vital sostener su recuerdo y sobre todo su pensamiento. Este último de forma prioritaria en consecuencia con la incesante labor que Facio hizo desde su juventud en la apertura de nuevas ideas en la mesa del debate nacional. Asimismo, nos es imperante esta serie de reflexiones en la actualidad, ya que aunque han pasado los años, la inclusión de las juventudes en la dirección del país sigue siendo deficiente en nuestro país. 
Seguir el ejemplo de Rodrigo Facio no es solo un orgullo, también significa una lucha enorme contra la cultura del olvido que tenemos en nuestro país, al considerar que lo nuestro es inferior; de lo contrario, el producto social que vamos construyendo amerita cada vez más que nos recordemos entre nosotros y nunca olvidemos que somos de la misma parcela de tierra, que el humanismo si bien nos une como humanidad, no debemos permitir que nos separe entre quienes habitamos en la Costa Rica que vivió Rodrigo Facio y ahora nosotros proseguimos en el devenir de los tiempos.

\section{REFERENCIAS}

Castro, O. (2003). Rodrigo Facio en la Constituyente de 1949. San José. Costa Rica: EUNED.

Cruz, R. C. (1960). La Conferencia del Rector Facio sobre Marxismo. Una Respuesta. Revista de la Universidad de Costa Rica, 24-123.

Fallas, A. S. (2012). El Papel de Rodrigo Facio en la Constituyente de 1949. Revista de Ciencias Sociales 130, 53-60.
Facio Brenes, R. (enero-junio de 1957). Dos palabras sobre la revista de Filosofía de la Universidad de Costa Rica. Revista de Filosofía de la Universidad de Costa Rica 1(1), 3-6.

Facio Brenes, R. (1961). Palabras de Despedida del Dr. Rodrigo Facio de la Rectoría, Universidad de Costa Rica [Grabado por A. d. (UCR)]. San Pedro, San José, Costa Rica.

Hess E, R. (1972). Rodrigo Facio. El Economista. Serie Económica y Estadística 36. San José, Costa Rica: Publicaciones de la Universidad de Costa Rica.

Oduber Q. D. (1994). Raíces del Partido Liberación Nacional: Notas para una Evaluación Histórica. San José, Costa Rica: Editorial Universidad Estatal a Distancia (euned).

Fecha de ingreso: $31 / 10 / 2017$ Fecha de aprobación: 06/03/2018 